\title{
Stable Bloch Oscillations of Cold Atoms with Time-Dependent Interaction
}

\author{
C. Gaul, ${ }^{1}$ R. P. A. Lima, ${ }^{2}$ E. Díaz, ${ }^{2}$ C. A. Müller,${ }^{1,3}$ and F. Domínguez-Adame ${ }^{2}$ \\ ${ }^{1}$ Physikalisches Institut, Universität Bayreuth, D-95440 Bayreuth, Germany \\ ${ }^{2}$ GISC, Departamento de Física de Materiales, Universidad Complutense, E-28040 Madrid, Spain \\ ${ }^{3}$ Laboratoire Kastler-Brossel, UPMC, ENS, CNRS; 4 Place Jussieu, F-75005 Paris, France \\ (Received 1 March 2009; revised manuscript received 4 June 2009; published 26 June 2009)
}

\begin{abstract}
We investigate Bloch oscillations of interacting cold atoms in a mean-field framework. In general, atom-atom interaction causes dephasing and destroys Bloch oscillations. Here we show that Bloch oscillations are persistent if the interaction is modulated harmonically with suitable frequency and phase. For other modulations, Bloch oscillations are rapidly damped. We explain this behavior in terms of collective coordinates whose Hamiltonian dynamics permits one to predict a whole family of stable solutions. In order to describe also the unstable cases, we carry out a stability analysis for Bogoliubov excitations. Using Floquet theory, we are able to predict the unstable modes as well as their growth rate, found to be in excellent agreement with numerical simulations.
\end{abstract}

DOI: 10.1103/PhysRevLett.102.255303

The dynamics of quantum particles in periodic potentials subjected to uniform acceleration has a long history and fascinating physics. Electrons in tilted periodic potentials present dynamical localization and may undergo coherent oscillations, both in real and in momentum space, known as Bloch oscillations (BOs) [1]. BOs were observed for the first time as coherent oscillations of electronic wave packets in semiconductor superlattices [2,3]. These oscillations are due to interference of partially scattered wave amplitudes and therefore can be observed for any phasecoherent waves accelerated in periodic potentials. BOs have been directly observed with ultracold atoms [4,5], Bose-Einstein condensates [6], and noninteracting fermions [7] in tilted optical lattices.

After excitation, BOs persist until the quantum particles lose their phase coherence. In Bose-Einstein condensates, interatomic interactions lead to a rapid broadening of the momentum distribution and a strong dephasing. Even in the most favorable experimental conditions, only a few cycles are usually observed. It is then believed that nonlinearities must generally lead to a breakdown of the BOs [8-10].

In cold-atom experiments, one is able to change the interaction strength by means of Feshbach resonances $[11,12]$. Reducing the interaction to zero, Gustavsson et al. [13] were able to increase the dephasing time of a cloud of Cs atoms from a value slightly larger than a Bloch period $T_{B}$ to more than $2 \times 10^{4} T_{B}$. A different way of obtaining stable Bloch oscillations was proposed by Salerno, Konotop, and Bludov [14]: Properly designing the spatial dependence of the scattering length around the zero crossing in a Feshbach resonance should result in a long-living BO of bright solitons. In this Letter, we propose instead to modulate the interaction harmonically in time by an oscillating, but spatially homogeneous, magnetic field close to a Feshbach resonance [15], which should be much easier experimentally. Although, in general, atom-atom
PACS numbers: 67.85.-d, 03.75.Lm, 37.10.Jk, 52.35.Mw

interactions lead to a rapid dephasing of BOs, remarkably with some harmonic modulations we find stable oscillations of the condensate [see Fig. 1(a)], while for others the oscillations are rapidly dephased [see Fig. 1(b)].

Our starting point for this study is the mean-field GrossPitaevskii equation for the discrete tight-binding model [16]

$$
i \dot{\Psi}_{n}=-\Psi_{n+1}-\Psi_{n-1}+F n \Psi_{n}+g(t)\left|\Psi_{n}\right|^{2} \Psi_{n},
$$

where $\Psi_{n}$ denotes the wave function at each potential well $n$ of the optical lattice. The overdot indicates the time derivative, the hopping parameter is set as the unit of energy, and $\hbar=1$. We assume that initially the atomic cloud has a Gaussian density profile centered at rest in the lattice: $\Psi_{n}(0)=\left(2 \pi \sigma_{0}^{2}\right)^{-1 / 4} \exp \left[-n^{2} / 4 \sigma_{0}^{2}\right]$. In the noninteracting case $g(t)=0$, the atomic cloud oscillates with the Bloch frequency $\omega_{B}=2 \pi / T_{B}=F$.

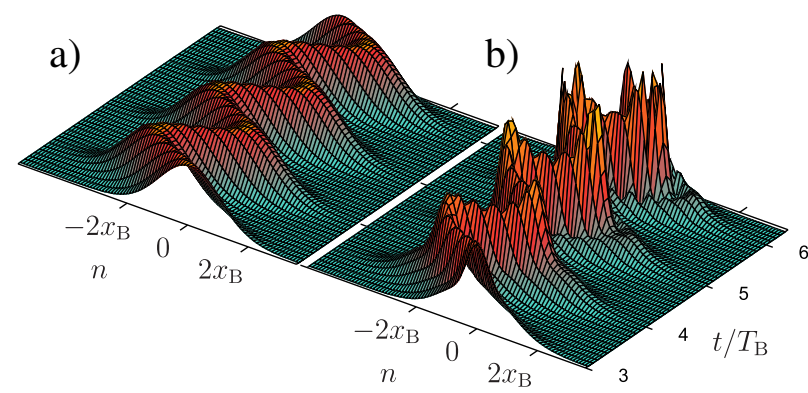

FIG. 1 (color online). Condensate density $\left|\Psi_{n}(t)\right|^{2}$ for two interactions modulated harmonically at the Bloch frequency $\omega_{B}=F$ : (a) $g(t)=g_{0} \cos (F t)$ and (b) $g(t)=g_{0} \sin (F t)$, with $F=0.2$ and $g_{0}=1$. Data from numerical integration of the Gross-Pitaevskii equation (1). The initial width of the wave packet is $\sigma_{0}=10$, equal to the amplitude $x_{B}=2 / F$ of free BOs. While the BOs in (a) are perfectly stable, they are rapidly destroyed in (b). 
To investigate the interacting case, we first solve (1) numerically by means of the fourth-order Runge-Kutta method. Figure 1 shows the time evolution of the condensate density $\left|\Psi_{n}(t)\right|^{2}$ for two different harmonic interactions modulated at the Bloch frequency. For $g(t)=$ $g_{0} \cos (F t)$, we observe that the initial Gaussian shape is preserved over time, and the condensate performs perfectly stable BOs with frequency $\omega_{B}$. On the contrary, for $g(t)=$ $g_{0} \sin (F t)$, the initial shape is distorted already after a few Bloch cycles. As a consequence, BOs are rapidly damped.

In order to understand how the interaction affects BOs of a Gaussian wave packet, we start with a description in terms of collective coordinates [9]. As free variables, we chose the center-of-mass position $x(t)=\langle n\rangle=$ $\sum_{n} n\left|\Psi_{n}(t)\right|^{2}$ and width $w(t)=\left\langle[n-x(t)]^{2}\right\rangle$ and their conjugate momenta $p(t)$ and $b(t)$, respectively. These are defined by their generating rôle $-i \partial_{p} \Psi_{n}=n \Psi_{n}$ and similarly for $b$. The wave function is up to a global phase parametrized as

$$
\Psi_{n}(t)=e^{i p(t) n} A(n-x(t), w(t), b(t)),
$$

where the rapidly varying exponential $\exp [i p(t) n]$ is factorized from a smooth Gaussian envelope

$$
A(n, w, b)=\frac{1}{(2 \pi w)^{1 / 4}} \exp \left[-\frac{n^{2}}{4 w}+i b n^{2}\right] .
$$

The equation of motion (1) derives as $i \dot{\Psi}_{n}=\partial H / \partial \Psi_{n}^{*}$ from the nonlinear Hamiltonian

$$
H=\sum_{n}\left\{-\left(\Psi_{n+1} \Psi_{n}^{*}+\text { c.c. }\right)+F n\left|\Psi_{n}\right|^{2}+\frac{g(t)}{2}\left|\Psi_{n}\right|^{4}\right\} .
$$

Inserting the ansatz (2), Taylor-expanding the discrete gradient to second order, and performing the Gaussian integration, we find the effective Hamiltonian

$$
H_{\mathrm{cc}}=F x-\left(2-\frac{1+16 b^{2} w^{2}}{4 w}\right) \cos p+\frac{g(t)}{4 \sqrt{\pi}} w^{-1 / 2} .
$$

By construction, the collective coordinates obey the canonical equations of motion

$$
\begin{aligned}
\dot{p} & =-\frac{\partial H_{\mathrm{cc}}}{\partial x}=-F, \\
\dot{x} & =\frac{\partial H_{\mathrm{cc}}}{\partial p}=\left(2-\frac{1+16 b^{2} w^{2}}{4 w}\right) \sin p, \\
\dot{b} & =-\frac{\partial H_{\mathrm{cc}}}{\partial w}=\frac{1-16 w^{2} b^{2}}{4 w^{2}} \cos p+\frac{g(t)}{8 \sqrt{\pi}} w^{-3 / 2}, \\
\dot{w} & =\frac{\partial H_{\mathrm{cc}}}{\partial b}=8 w b \cos p .
\end{aligned}
$$

The initial conditions for the case under study are $x(0)=0$, $p(0)=0, w(0)=\sigma_{0}^{2}$, and $b(0)=0$. Equation (6) shows that within this ansatz, the momentum of the centroid is always given as $p(t)=-F t$, which then serves as the driving term in the other equations. The autonomous equations (6c) and (6d) describing the width can be solved first, their solution finally conditioning the centroid motion obtained by integrating (6b).

Let us study the implications of these equations in some simple cases. The complex width of the Gaussian envelope $z=\left[w^{-1}-4 i b\right]^{-1}$ evolves according to

$$
\dot{z}=i \cos F t+\frac{i g(t)}{2 \sqrt{\pi}} \frac{z^{2}}{|z|^{3}}(\operatorname{Re} z)^{3 / 2} .
$$

In the linear case $g=0$, this equation has the exact solution $\operatorname{Re} z_{0}=w(0)=\sigma_{0}^{2}$ and $\operatorname{Im} z_{0}(t)=F^{-1} \sin F t$. It describes a Gaussian wave packet with a breathing width $w_{0}(t)=\sigma_{0}^{2}\left[1+(\sin F t)^{2} /\left(F \sigma_{0}^{2}\right)^{2}\right]$. This solution is valid for small $1 /\left(F \sigma_{0}^{2}\right)$, i.e., sufficiently broad wave packets. Within the collective-variable approach, the amplitude of BOs is actually determined by $R:=(8 \operatorname{Re} z)^{-1}=$ $\left(1+16 b^{2} w^{2}\right) /(8 w)$, appearing in $(6 \mathrm{~b})$. In the linear case, its constant value $R_{0}=\sigma_{0}^{-2} / 8 \ll 1$ yields the usual BOs $x_{0}(t)=x_{B} \cos F t$ with amplitude $x_{B}=2\left(1-R_{0}\right) / F$.

A constant nonlinearity $g(t)=g_{0}$ leads to a damping of the oscillation amplitude. With the highly nonlinear and rapidly oscillating equations of motion (6c) and (6d), one finds $\ddot{R}=g_{0} \mathcal{W}(t) \cos F t+g_{0}^{2} /\left[16 \pi w(t)^{2}\right]$. The first term contains contributions from $w(t)$ and $b(t)$ that are very effectively suppressed over a Bloch cycle by the oscillating factor $\cos F t$. The decay to lowest order in $g_{0}$ therefore is driven by the second term, and the amplitude decreases initially like $x_{B}(t)=(2 / F)\left(1-R_{0}-2 g_{0}^{2} R_{0}^{2} t^{2} / \pi\right)[9,10]$.

Let us now consider a time-dependent interaction of the form $g(t)=g_{0} \sin (\omega t+\delta)$. The equations of motion for the collective coordinates allow one to identify a whole family of values $(\omega, \delta)$ that result in strictly periodic motion. Formally, we can combine (6c) and (6d) to a single equation $\dot{v}=X(v, t)$ for $v=(w, b)$. This Hamiltonian flow is driven by a vector field $X(\cdot, t)$ that depends explicitly on $\cos F t$ and $g(t)$. Let now $\omega=\left(\nu_{1} / \nu_{2}\right) \omega_{B}$, with $\nu_{1}, \nu_{2} \in \mathbb{N}$, be commensurate with the Bloch frequency $\omega_{B}=F$. Then $X(\cdot, t+T)=X(\cdot, t)$ is periodic with $T=$ $\nu_{2} T_{B}$. Since different trajectories $v(t)$ cannot intersect, the flow dynamics is strictly periodic if there exists a time $\tau$ such that $v(\tau-T / 2)=v(\tau+T / 2)$. This in turn is guaranteed by the equation of motion if $X(\cdot, t)$ is odd around $\tau$ : $X(\cdot, \tau-t)=-X(\cdot, \tau+t)$. But since $X$ contains $\cos F t$, this time can only be one of its zeros $\tau_{j}=(2 j+1) \times$ $\frac{\pi}{2} F^{-1}, j \in \mathbb{N}$. This then requires also the interaction to be odd at that point, which fixes $\delta_{j}=-\omega \tau_{j}$. Therefore, the family of time-dependent interactions with periodic solutions contains all linear combinations of

$$
g(t)=g_{0} \sin \left(\frac{\nu_{1}}{\nu_{2}}\left[F t-\frac{\pi}{2}(2 j+1)\right]\right), \quad \nu_{1}, \nu_{2}, j \in \mathbb{N},
$$

for example, $\cos [(2 n+1) F t], \sin (2 n F t), \cos (F t / 3)$, or $\sin (2 F t / 3)$. In all such cases, the periodicity of $w(t)$ and $b(t)$ predicts, via $(6 \mathrm{~b})$, perfect BOs in spite of the underlying nonlinearity. 
This stabilization mechanism does not rely on suppressing the nonlinearity at the band edges, which can, in general, be helpful to avoid Landau-Zener tunneling [16] but is irrelevant in our single-band description. Instead, the simplest stable interaction of type $g(t)= \pm \cos (F t)=$ $\pm \cos [p(t)]$ has its maximum amplitude with a positive (negative) sign at the band center $p(t)=0$ and a negative (positive) sign at the band edge $p(t)=\pi$. Clearly, it does not vanish at the band edge but rather at the band midpoint, where the mass changes sign and dynamical instabilities would start to appear $[8,17]$.

We have tested the collective-variable predictions by comparing the centroid $x(t)$ as obtained from Eq. (6) with the results from the numerical integration of (1). Collective coordinates provide an excellent description of long-living BOs [Fig. 2(a)]. In the damped case $g(t)=$ $g_{0} \sin (F t)$, this approach also predicts a breakdown of BOs, but not quite correctly [Fig. 2(b)]. This ansatz allows only the Gaussian width to respond to the nonlinear perturbation, but it cannot capture the actual dephasing mechanism, which involves the excitation of fluctuations as apparent from Fig. 1(b).

To proceed beyond collective coordinates we start with an infinitely wide wave packet, namely, an atomic cloud with uniform density $\left|\Psi_{n}^{0}\right|^{2}=\rho_{0}$. In this case, Eq. (1) is exactly solved by $\Psi_{n}^{0}(t)=\sqrt{\rho_{0}} \exp [-i \phi(t)]$, with

$$
\phi(t)=F n t-\frac{2}{F} \sin (F t)+\int_{0}^{t} d t^{\prime} \mu\left(t^{\prime}\right)
$$

in terms of the chemical potential $\mu(t)=\rho_{0} g(t)$. This

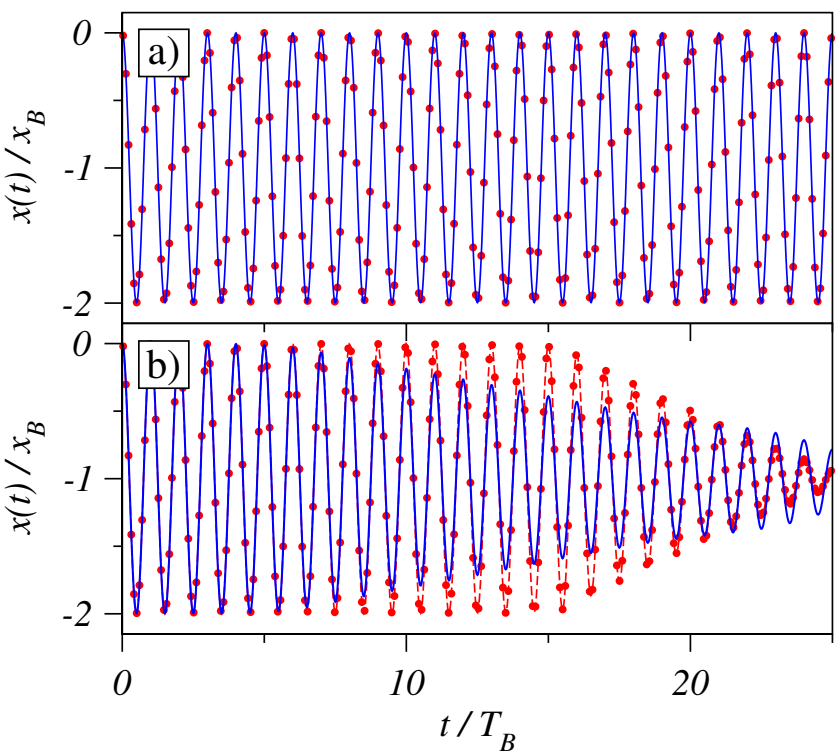

FIG. 2 (color online). Centroid $x(t)$ for (a) $g(t)=g_{0} \cos (\omega t)$ and (b) $g(t)=g_{0} \sin (\omega t)$, with $\omega=F=0.2$ and $g_{0}=1.0$. The solid line is obtained by numerical integration of (1); circles connected by dashed lines are the solution of the collectivecoordinate Eqs. (6a)-(6c). In the stable case (a) both curves agree, whereas in (b) the collective coordinates predict a later onset of damping. solution predicts that an infinitely narrow momentum distribution will perfectly Bloch oscillate in momentum space; i.e., the condensate current will oscillate homogeneously without signatures in position space, and this for arbitrary interaction.

In the following, we study the broadening of the momentum distribution due to the growth of small perturbations. If the wave function deviates slightly from the homogenous solution $\Psi_{n}(t)=\left[\sqrt{\rho_{0}}+\Phi(n, t)\right] \times$ $\exp [-i \varphi(t)]$, linearizing (1) gives the equation of motion for the fluctuation $\Phi(n, t)$ :

$$
i \dot{\Phi}=-\cos (F t) \Phi^{\prime \prime}+2 i \sin (F t) \Phi^{\prime}+2 \mu(t) \operatorname{Re} \Phi .
$$

$\Phi(n, t)$ is assumed to be a smooth function with spatial derivative $\Phi^{\prime}=\partial_{n} \Phi$. The interaction term acts on $\operatorname{Re} \Phi$, i.e., the density fluctuation. Separating real and imaginary parts $\Phi(n, t)=s(m, t)+i d(m, t)$ while transforming to the reference frame $m=n-x_{0}(t)$ of the unperturbed BO with $x_{0}(t)=2 F^{-1} \cos F t$ and going to the momentum representation brings (10) to

$$
\begin{aligned}
& \dot{d}_{k}=-\left[k^{2} \cos (F t)+2 \mu(t)\right] s_{k}, \\
& \dot{s}_{k}=k^{2} \cos (F t) d_{k} .
\end{aligned}
$$

These are the Bogoliubov-de Gennes equations for excitations of a homogeneous condensate with chemical potential $\mu(t)$ and a time-dependent mass, such that $\epsilon_{k}^{0}(t)=k^{2} \cos (F t)$. The width of the momentumspace distribution $(\Delta k)^{2}=\left(N \rho_{0}\right)^{-1} \sum_{k}(k-p)^{2}\left|\Psi_{k}\right|^{2}=$ $\left(N \rho_{0}\right)^{-1} \sum_{k} k^{2}\left(\left|d_{k}\right|^{2}+\left|s_{k}\right|^{2}\right)$ is given by these amplitudes. Thus, their stability is the key to understand the stability of BOs.

In Eqs. (11), we have again the structure of a first-order Hamiltonian equation of motion (now with a linear evolution operator) depending on $\cos (F t)$ and $\mu(t)=\rho_{0} g(t)$. Thus the same argumentation as for the collective coordinates holds: Solutions are periodic, and hence perturbations do not grow if $g(t)$ is of the type (8).

But now we are also able to make quantitative predictions about both stable and unstable cases. Thanks to the linearity of (11), Floquet theory [18] applies if the interaction is modulated at a commensurate frequency $\omega=$ $\left(\nu_{1} / \nu_{2}\right) \omega_{B}$. The stability of this system can be assessed by integrating the differential equations (11) over one period $T=\nu_{2} T_{B}$ with two different initial conditions: $\left\{d_{k}^{1}(0)=1, s_{k}^{1}(0)=0\right\}$ and $\left\{d_{k}^{2}(0)=0, s_{k}^{2}(0)=1\right\}$. A perturbation with wave vector $k$ is stable when $\left|\Delta_{k}\right| \leq 1$, where $\Delta_{k}=(1 / 2)\left[d_{k}^{1}(T)+s_{k}^{2}(T)\right]$. In the periodic cases, $\Delta_{k}=1$. An unstable fluctuation with wave vector $k$ grows exponentially with the Lyapunov exponent $\lambda_{k}=$ $T^{-1} \log \left[\Delta_{k}+\sqrt{\Delta_{k}^{2}-1}\right]$.

Figure 3 indicates by black squares those parameters $\left(\omega / \omega_{B}, k\right)$ in the case $g(t)=g_{0} \sin (\omega t)$, for which $\left|\Delta_{k}\right| \leq$ 1 assures stability. Full lines correspond to the stable cases at $\omega / \omega_{B}=2 n / 5, n \in \mathbb{N}$, predicted by (8); other stable lines are not resolved due to the finite frequency resolution 


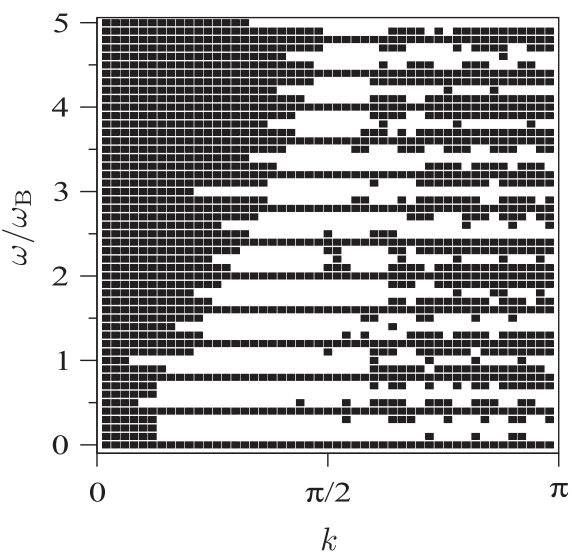

FIG. 3. Stability map for $g(t)=g_{0} \sin (\omega t)$, with $\omega_{B}=F=$ 0.2 and $g_{0}=1.0$. Black squares indicate the wave vectors $k$ of stable excitations at each frequency $\omega$. The stable cases at $\omega / \omega_{B}=2 n / 5$ predicted by (8) appear as full lines; the other stable lines are not resolved due to the finite frequency step 0.1 used here. For the case of $\cos (\omega t)$, the diagram is similar, with stable lines at $\omega / \omega_{B}=(2 n+1) / 5$.

0.1 used for the plot. For most frequencies, there are many $k$ vectors belonging to unstable excitations, so the $\mathrm{BO}$ will decay [Fig. 1(b)].

For a quantitative test of Floquet-theory predictions, we choose a larger width $\sigma_{0}=100$ and a weak interaction parameter $\mu_{0}=0.01$. As obvious from Fig. 3, unstable modes are typically encountered at intermediate values of $k$. Floquet analysis predicts as the most unstable mode $k \approx$ 0.44 with a Lyapunov exponent of $\lambda_{\max } \approx 0.1815 T_{B}^{-1}$. The numerically calculated centroid motion (similarly to Fig. 2) starts to get damped around $200 T_{B}$. The $k$-space picture (Fig. 4) is much more revealing and shows that the most unstable mode starts to grow much earlier. The numerically observed dominant mode and also its growth

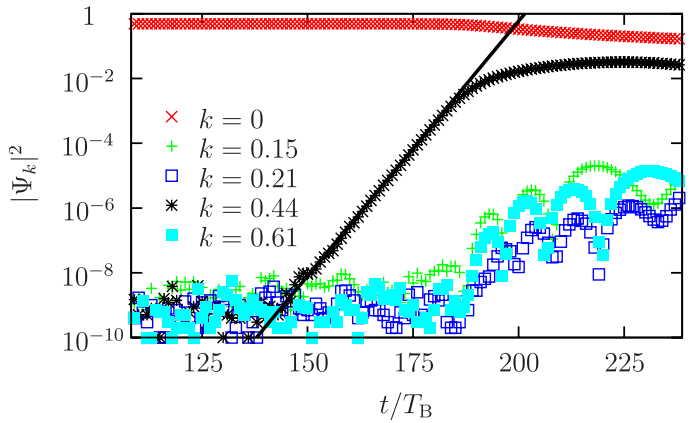

FIG. 4 (color online). Momentum density $\left|\Psi_{k}\right|^{2}$ for selected $k$ modes in the unstable case $g(t)=g_{0} \sin (F t)$. The original wave function is centered around $k=0$. The solid line marks the growth rate of the most unstable mode as predicted by Floquet theory. The growth of this mode precedes the damping of the centroid motion that sets in at $t \approx 200 T_{B}$. Numerical parameters: $\sigma_{0}=100, \mu_{0}=0.01$, and $F=0.2$. rate are in perfect agreement with our Floquet-theory predictions.

In conclusion, we have shown that stable BOs of cold atoms are possible when the atom-atom interaction is modulated in time with suitable frequency and phase. Collective coordinates are found to accurately describe undamped BOs. For commensurate frequencies $\omega$ and $\omega_{B}$, we have identified a class of time-dependent interactions, for which the BOs remain perfectly periodic. But collective coordinates cannot capture the dynamics of unstable cases where the main wave packet decays by radiating excitations. In order to explain the dephasing of the oscillations in unstable cases, we applied a stability analysis based on Floquet theory that is in excellent agreement with the numerical results. Using harmonically modulated interaction thus opens the possibility of studying the precise influence of other dephasing mechanisms on Bloch oscillations, such as deliberately introduced disorder [19].

Travel between Bayreuth and Madrid is supported by the DAAD-MEC joint program Acciones Integradas. Work at Madrid was supported by MEC (Project MOSAICO) and BSCH-UCM (Project No. PR34/07-15916). R. P. A. L. acknowledges support by MEC through the Juan de la Cierva program and G. Rowlands for helpful discussions. C. G. acknowledges support by DFG and DAAD, and C.M. acknowledges helpful discussions with Y. Gaididei.

[1] F. Bloch, Z. Phys. 52, 555 (1929).

[2] J. Feldmann et al., Phys. Rev. B 46, R7252 (1992).

[3] K. Leo et al., Solid State Commun. 84, 943 (1992).

[4] M. BenDahan, E. Peik, J. Reichel, Y. Castin, and C. Salomon, Phys. Rev. Lett. 76, 4508 (1996).

[5] S. R. Wilkinson, C.F. Bharucha, K. W. Madison, Q. Niu, and M. G. Raizen, Phys. Rev. Lett. 76, 4512 (1996).

[6] B. P. Anderson and M. A. Kasevich, Science 282, 1686 (1998).

[7] G. Roati et al., Phys. Rev. Lett. 92, 230402 (2004).

[8] L. Fallani et al., Phys. Rev. Lett. 93, 140406 (2004).

[9] A. Trombettoni and A. Smerzi, Phys. Rev. Lett. 86, 2353 (2001).

[10] D. Witthaut, M. Werder, S. Mossmann, and H. J. Korsch, Phys. Rev. E 71, 036625 (2005).

[11] E. A. Donley et al., Nature (London) 412, 295 (2001).

[12] M. Fattori et al., Phys. Rev. Lett. 100, 080405 (2008).

[13] M. Gustavsson et al., Phys. Rev. Lett. 100, 080404 (2008).

[14] M. Salerno, V. V. Konotop, and Yu. V. Bludov, Phys. Rev. Lett. 101, 030405 (2008).

[15] F. Kh. Abdullaev, E. N. Tsoy, B. A. Malomed, and R. A. Kraenkel, Phys. Rev. A 68, 053606 (2003).

[16] O. Morsch and M. Oberthaler, Rev. Mod. Phys. 78, 179 (2006).

[17] B. Wu and Q. Niu, New J. Phys. 5, 104 (2003).

[18] G. Teschl, Ordinary Differential Equations and Dynamical Systems (unpublished), available at http://www.mat. univie.ac.at/gerald/ftp/book-ode/.

[19] S. Drenkelforth et al., New J. Phys. 10, 045027 (2008). 